\title{
Bioinspired metaheuristics for image segmentation
}

\author{
Valentín Osuna-Enciso \\ Centro de Investigación en Computación-IPN, Av. Juan de Dios Batiz $S / N$, \\ Col. Nueva Industrial Vallejo, México, D.F., México \\ Advisors: Erik Cuevas, Humberto Sossa \\ Date and location of the PhD thesis defense: 2nd December 2013, \\ Centro de Investigación en Computación - Instituto Politécnico Nacional \\ Received 4th Dec 2013; accepted 25th May 2014
}

\section{Abstract}

In general, the purpose of Global Optimization (GO) is finding the global optimum of an objective function defined inside a search space. The GO has applications in many areas of science, engineering, economics, among other, where mathematical models are utilized. Those algorithms are divided into two groups: deterministic, and evolutionary. Since deterministic methods only provide a theoretical guarantee of locating local minimums of the objective function, they face great difficulties in solving GO problems. On the other hand, evolutionary methods are faster in locating a global optimum than deterministic ones, because they operate over a population of candidate solutions, therefore they have a bigger likelihood of finding the global optimum, and a better adaptation to black box formulations or complicated function forms.

Despite that during the last decade the field of metaheuristics applied to optimization has had an important increase, the quest of such methods still considered as an open problem in research, due to the most part that they yet present difficulties; for instance, the premature convergence, and the difficulty to overcome local optimum values in multimodal functions. For that reason, in this work it is proposed a bio-inspired algorithm, which utilizes the allostatic mechanism as a base model.

Allostasis means 'to maintain stability through change (of several set points -SP)'. That medical model considers the existence of several set points of mechanisms, their non-linear relationships with mediators, other mechanisms, and the brain. Moreover, in this model the brain 'predicts' the new set points, allowing faster responses concerning to the instability. In general terms, once the brain detects some external or internal change (stress, pollution, changes in social status, disease, etc), it determines if the stability (of a single organ, organ system, condition, health, etc) is compromised. Supposing that the instability is confirmed, then the body activates the communication-coordination scheme and starts sending chemical-electrical signals (mediators) to specific mechanisms (viz., target cells, tissues, organs, or even other OS). Those mechanisms should modify

Correspondence to: $<$ valentin.osuna@cucei.udg.mx $>$

Recommended for acceptance by $<$ Alicia Fornés and Volkmar Frinken $>$

ELCVIA ISSN: $1577-5097$

Published by Computer Vision Center / Universitat Autonoma de Barcelona, Barcelona, Spain 
their behavior (as a result of adjusting their functioning SP), and send back a feedback signal to the brain, which evaluates whether the changes are good enough to bring back the stability, in whose case, the modified SP will replace the previous, and these will kept in memory.

By using the allostatic mechanisms as a metaphor, it is proposed a metaheuristic algorithm, which we called Allostatic Optimization (AO). The AO algorithm provides a searching procedure that is population-based, in which all the individuals, seen as SP, are defined in a multidimensional search space; aforementioned agents are either generated or modified by mean of several evolutionary operators that emulate different operations used by the allostatic process, whereas an objective function evaluates the individual stability of each SP.

We made a comparison of $\mathrm{AO}$ against $\mathrm{DE}, \mathrm{ABC}$ and $\mathrm{PSO}$ and, we found that the proposed algorithm favors the exploration process, and eliminates some flaws related with the premature convergence, this is because $\mathrm{AO}$ is capable to maintain the population diversity; in fact, in the $57 \%$ of the functions, the diversity maintained by AO relieves the convergence, by introducing operators which avoid particle concentration on some search space regions, and so helping the exploration. Neverheless, it was also found that maintaining a high population diversity does not guarantee a proper convergence of $\mathrm{AO}$ in all the benchmark functions (43\%), thus a potential investigation work could be to perform a more complete study of the relations among the function properties, the diversity, and an adequate algorithm convergence.

With the idea of demonstrating the utility of the algorithm in a particular family of problems, AO was utilized in image segmentation by means of a mixture of functions. One way to achieve segmentation is by utilizing thresholding selection, where each pixel that belongs to a class is labeled according to a selected threshold, giving as a result pixel groups which share visual characteristics in the image. In this work it was utilized a method based on a mixture of Cauchy functions, to approximate gray level histograms of images taken from known benchmarks, and it was found that $\mathrm{AO}$ improves the segmentation quality in about $14 \%$, compared with Otsu's method.

Moreover, the metaheuristic algorithms DE, ABC and PSO were examined in contrast they are applied to image segmentation by using a method that uses a mixture of Gaussian functions to approximate 1D histograms, since an analysis of that type was not found in the literature; in the empirical results, DE gave best results related with convergence speed, as well as the segmentation quality, when they are compared with ground-truth images.

\section{References}

[1] M. Pardalos Panos, H. Romeijn Edwin, Hoang Tuy, Recent developments and trends in global optimization, Journal of Computational and Applied Mathematics 124 (2000) 209-228.

[2] Floudas, C., Akrotirianakis, I., Caratzoulas, S., Meyer, C., Kallrath, J. Global optimization in the 21st century: Advances and challenges. Computers \& Chemical Engineering, 29(6), (2005), 1185-1202.

[3] Ying, J., Ke-Cun, Z., Shao-Jian, Q., A deterministic global optimization algorithm. Applied Mathematics and Computation, 185(1), (2007), 382-387.

[4] Georgieva, A., Jordanov, I., Global optimization based on novel heuristics, low-discrepancy sequences and genetic algorithms. European Journal of Operational Research, 196, (2009), 413-422.

[5] Lera, D., Sergeyev, Ya. Lipschitz and Hölder global optimization using space-filling curves. Applied Numerical Mathematics, 60(1-2), (2010), 115-129.

[6] J. Kennedy, R.C. Eberhart, Particle swarm optimization, in: Proceedings of the 1995 IEEE International Conference on Neural Networks, vol. 4, 1995, pp. 1942-1948.

[7] D. Karaboga, An idea based on honey bee swarm for numerical optimization, Technical report,-TR06, Erciyes University, Engineering Faculty, Computer Engineering Department, 2005. 
[8] Dorigo, M., Maniezzo, V., Colorni, A., 1991. Positive feedback as a search strategy. Technical Report No. 91016, Politecnico di Milano.

[9] S. Kirkpatrick, C. Gelatt, M. Vecchi, Optimization by simulated annealing, Science 220 (4598) (1983) 671-680.

[10] B. İlker, S. Birbil, F. Shu-Cherng, An Electromagnetism-like Mechanism for Global Optimization. Journal of Global Optimization, 25 (2003) 263-282.

[11] E. Rashedia, H. Nezamabadi-pour, S. Saryazdi. Filter modeling using Gravitational Search Algorithm. Engineering Applications of Artificial Intelligence, 24(1), 2011, 117-122.

[12] Z.W. Geem, J.H. Kim, G.V. Loganathan, A new heuristic optimization algorithm: harmony search, Simulation 76 (2) (2001) 60-68.

[13] L.J. Fogel, A.J. Owens, M.J. Walsh, Artificial Intelligence through Simulated Evolution, John Wiley, Chichester, UK, 1966.

[14] K. De Jong, Analysis of the behavior of a class of genetic adaptive systems, Ph.D. Thesis, University of Michigan, Ann Arbor, MI, 1975.

[15] J.R. Koza, Genetic programming: a paradigm for genetically breeding populations of computer programs to solve problems, Rep. No. STAN-CS-90-1314, Stanford University, CA, 1990.

[16] J.H. Holland, Adaptation in Natural and Artificial Systems, University of Michigan Press, Ann Arbor, MI, 1975.

[17] de Castro LN, Von Zuben FJ. Artificial immune systems: Part I - basic theory and applications. Technical report, TR-DCA 01/99. December 1999.

[18] Storn, R. and Price, K., Differential Evolution - A Simple and Efficient Heuristicfor Global Optimization over Continuous Spaces, Journal of Global Optimization,11(4), pp. 341-359, 1995.

[19] Norouzzadeh MS, Ahmadzadeh MR, Palhang M (2012) LADPSO: using fuzzy logic to conduct PSO algorithm. Appl Intell 37(2):290-304.

[20] Ben Ali YM (2012) Psychological model of particle swarm optimization based multiple emotions. Appl Intell 36(3):649-663.

[21] Manuel Martinez-Lavin, Angelica Vargas. Complex Adaptive Systems Allostasis in Fibromyalgia, Rheumatic Disease Clinics of North America, Volume 35, Issue 2, May 2009, Pages 285-298.

[22] Fletcher, J. M. Homeostasis as an explanatory principle in psychology, Psychological Review, Vol 49(1), Jan 1942, 80-87.

[23] Cannon, W.B., Bodily changes in pain, hunger, fear and rage: An account of recent researchers into the function of emotional excitement, 2nd edn, New York, Appleton, 1929.

[24] Cannon, W.B., The wisdom of the body, New York, W.W. Norton, 1932.

[25] Gross CG., Claude Bernard and the constancy of the internal environment, Neuroscientist, vol.4, pp. 380-385, 1988.

[26] Peter Sterling. Allostasis: A model of predictive regulation, Physiology \& Behavior, Volume 106, Issue 1, 12 April 2012, 5-15.

[27] L. Michael Romero, Molly J. Dickens, Nicole E. Cyr. The reactive scope model - A new model integrating homeostasis, allostasis, and stress, Hormones and Behavior, Volume 55, Issue 3, March 2009, 375-389.

[28] Bruce S McEwen, John C Wingfield. The concept of allostasis in biology and biomedicine, Hormones and Behavior, Volume 43, Issue 1, January 2003, Pages 2-15.

[29] Bruce S. McEwen. Allostasis and Allostatic Load: Implications for Neuropsychopharmacology, Neuropsychopharmacology, Volume 22, Issue 2, February 2000, Pages 108-124.

[30] Jay Schulkin. Allostasis: a neural behavioral perspective, Hormones and Behavior, Volume 43, Issue 1, January 2003, Pages 21-27.

[31] Yang X-S (2008) Nature-inspired metaheuristic algorithms. Luniver Press, Beckington.

[32] Chen DB, Zhao CX (2009) Particle swarm optimization with adaptive population size and its application. Appl Soft Comput 9(1):39-48.

[33] Erfu Yang, Nick H. Barton, Tughrul Arslan, Ahmet T. Erdogan, A novel shifting balance theory-based approach to optimization of an energy-constrained modulation scheme for wireless sensor networks. In Proceedings of the IEEE Congress on Evolutionary Computation, CEC 2008, June 1-6, 2008, Hong Kong, China. pages 2749-2756, IEEE, 2008.

[34] X. Duan, G. G. Wang, X. Kang, Q. Niu, G. Naterer, Q. Peng, Performance study of mode-pursuing sampling method, Engineering Optimization, Vol. 41, Iss. 1, 2009.

[35] Vesterstrom, J., Thomsen, R., A comparative study of differential evolution, particle swarm optimization, and evolutionary algorithms on numerical benchmark problems, Evolutionary Computation, 2004. CEC2004. Congress on, vol.2, no., pp. 1980- 1987 Vol.2, 19-23 June 2004.

[36] EfrénMezura-Montes, Jesús Velázquez-Reyes, and Carlos A. CoelloCoello, A comparative study of differential evolution variants for global optimization, In Proceedings of the 8th annual conference on Genetic and evolutionary computation (GECCO '06). ACM, New York, NY, USA, pp. 485-492, 2006. 